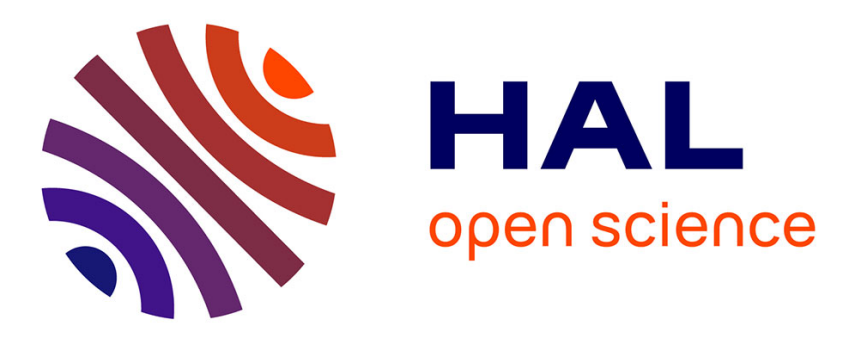

\title{
On disturbance decoupled observers for a class of bilinear systems
}

Michel Zasadzinski, Hugues Rafaralahy, Chokri Mechmeche, Mohamed

Darouach

\section{- To cite this version:}

Michel Zasadzinski, Hugues Rafaralahy, Chokri Mechmeche, Mohamed Darouach. On disturbance decoupled observers for a class of bilinear systems. Journal of Dynamic Systems, Measurement, and Control, 1998, 120 (3), pp.371-377. hal-00098140

\section{HAL Id: hal-00098140 \\ https://hal.science/hal-00098140}

Submitted on 24 Sep 2006

HAL is a multi-disciplinary open access archive for the deposit and dissemination of scientific research documents, whether they are published or not. The documents may come from teaching and research institutions in France or abroad, or from public or private research centers.
L'archive ouverte pluridisciplinaire HAL, est destinée au dépôt et à la diffusion de documents scientifiques de niveau recherche, publiés ou non, émanant des établissements d'enseignement et de recherche français ou étrangers, des laboratoires publics ou privés. 


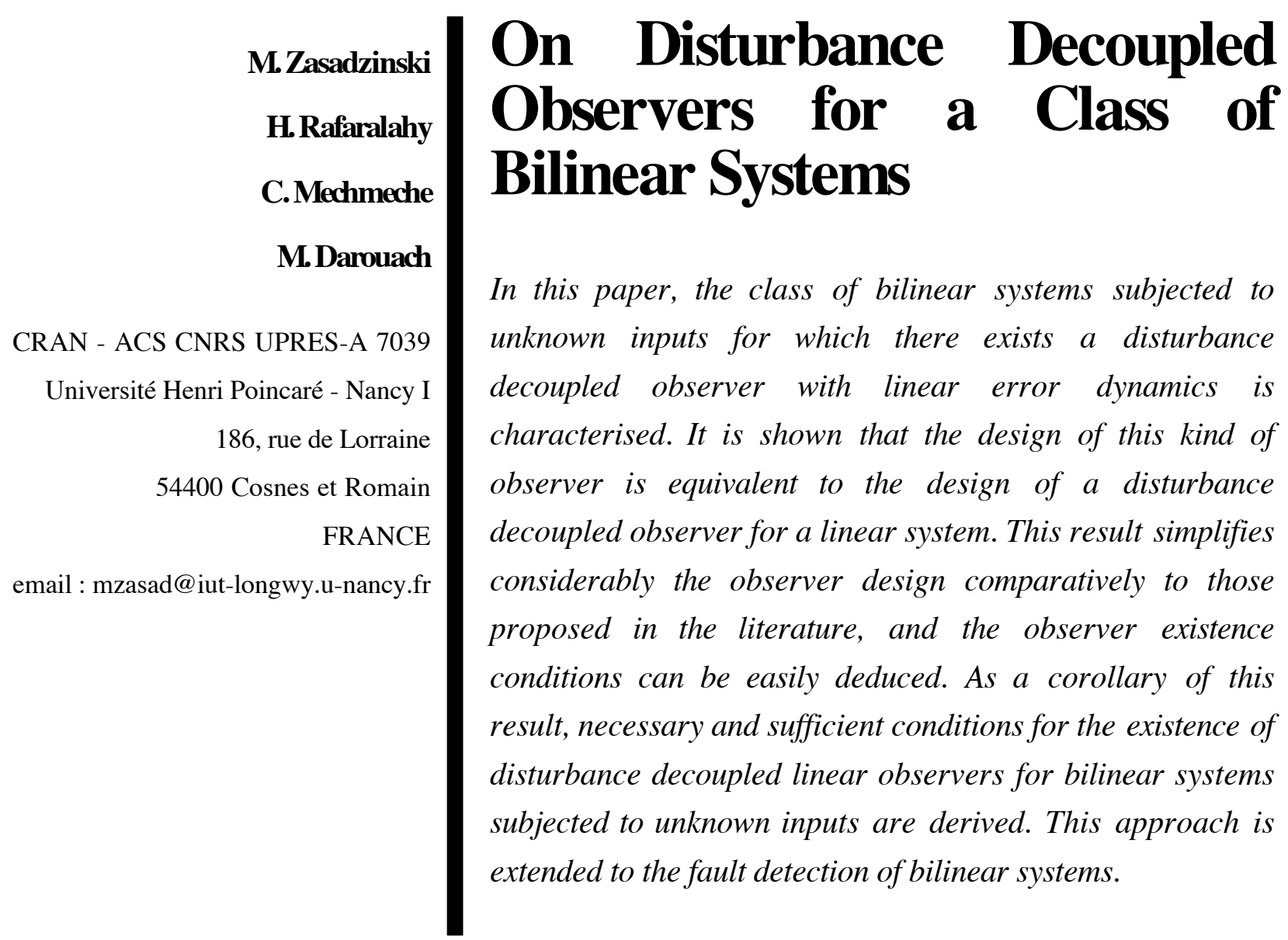

\section{Introduction}

Many important processes in engineering, biology, socio-economic and ecology may be modelled by bilinear systems when linear models are inadequate (Mohler, 1991). This is particularly true in modelling of biological (Williamson, 1977) and hydraulic processes (Hac, 1992).

The design of observers for bilinear systems have received more attention in the past years. As shown in Williamson (1977), the existence of observer for bilinear systems depends on the control inputs. Observers of bilinear systems are designed according to the requirement on the tracking error (Hara and Furuta, 1976; Grasselli and Isidori, 1981) or a performance index to be minimised (Bornard et al., 1988). The proposed observers in the literature fall into the following classes : observers requiring differentiators of the control input (Williamson, 1977), observers requiring on-line integration of some differential Riccati or Lyapunov matrix equation (Bornard et al., 1988), observers with input dependant error dynamics based on Lyapunov stability (Funahashi, 1979) and observers with linear error dynamics (Hara and Furuta, 1976). 
Like linear systems, bilinear ones are sometimes affected by time-varying unknown disturbances. Such unknown inputs can arise in the dynamical equation of the bilinear system due to actuator failures, plant disturbances, etc. Based on results in Hara and Furuta (1976), Hac (1992) and Saif (1993) considered the design of disturbance decoupled observers for bilinear systems with linear error dynamics.

This paper deals with the design of state observers for a class of bilinear systems affected by unmeasurable disturbances. It is shown that the design of a disturbance decoupled observer for a class of bilinear system can be reduced to the design of a disturbance decoupled observer for a corresponding linear system with additional virtual unknown inputs. This result simplifies significantly the observer design comparatively to those proposed by Hac (1992) and Saif (1993). Then conditions for the existence of this observer are given in terms of transmission zeros. This approach is extended to the design of linear disturbance decoupled observers for bilinear systems. The fault detection problem is treated by applying this approach to the design of disturbance decoupled residual observers.

This paper is organised as follows. The disturbance decoupled state observation problem for bilinear systems is formulated in Section 2. Section 3 is devoted to the analysis of the conditions for the existence of reduced-order and full-order disturbance decoupled observers for bilinear systems with linear error dynamics. Section 4 presents the characterisation of a class of bilinear systems subjected to unknown inputs for which there exists a linear disturbance decoupled observer and extends the results in Section 3 to the failure detection and isolation of bilinear systems. Finally in Section 5, the results are illustrated for a vehicle semi-active suspension and an electromechanical actuator.

\section{Problem Formulation}

Consider bilinear systems described by

$$
\begin{gathered}
\dot{\mathbf{x}}=\mathbf{A} \mathbf{x}+\sum_{i=1}^{m} \mathbf{D}_{i} u_{i} \mathbf{x}+\mathbf{B U}+\mathbf{F} \mathbf{d} \\
\mathbf{y}=\mathbf{C} \mathbf{x}+\mathbf{G d}
\end{gathered}
$$

where the state vector $\mathbf{x}(t) \in \mathbb{R}^{n}$, the control input $\mathbf{U}^{\mathrm{T}}(t)=\left[\begin{array}{lll}u_{1}(t) & \ldots & u_{m}(t)\end{array}\right] \in \mathbb{R}^{m}$, the unknown input vector $\mathbf{d}(t) \in \mathbb{R}^{q}$ and the output $\mathbf{y}(t) \in \mathbb{R}^{p}$. The aim of this paper is the analysis of the existence of an observer for the system (1)-(2) with the following structure

$$
\begin{gathered}
\dot{\mathbf{z}}=\mathbf{H z}+\mathbf{L y}+\mathbf{J U}+\sum_{i=1}^{m} \mathbf{N}_{i} u_{i} \mathbf{y} \\
\hat{\mathbf{x}}=\mathbf{M z}+\mathbf{P y}
\end{gathered}
$$

where $\mathbf{z}(t) \in \mathbb{R}^{j}(j \leq n)$ and $\hat{\mathbf{x}}(t) \in \mathbb{R}^{n}$. $\mathbf{H}, \mathbf{L}, \mathbf{N}_{i}, \mathbf{J}, \mathbf{M}$ and $\mathbf{P}$ are unknown matrices of appropriate dimensions. The observer (3)-(4) which is linear up to an output injection leads to the following definition (Hara and Furuta, 1976).

Definition 1. The system (3)-(4) is said to be a disturbance decoupled observer (DDO) of system (1)-(2) if and only if 


$$
\lim _{t \rightarrow \infty} \frac{\mathrm{d}^{k}}{\mathrm{dt}^{k}}(\mathbf{x}(t)-\hat{\mathbf{x}}(t))=\mathbf{0} \quad(k=0,1,2, \ldots)
$$

independently of $\mathbf{U}(t), \mathbf{d}(t), \mathbf{x}_{0}$ and $\mathbf{z}_{0}$.

In non-linear systems, specially in bilinear systems, there exist many kinds of observers, defined by a constraint on the observer error (Funahashi, 1979; Grasselli and Isidori, 1981; Hara and Furuta, 1976).

From definition 1, matrices $\mathbf{H}, \mathbf{L}, \mathbf{N}_{i}, \mathbf{J}, \mathbf{M}$ and $\mathbf{P}$ must be determined such that the state estimate $\hat{\mathbf{x}}(t)$ converges to $\mathbf{x}(t)$ asymptotically, without the knowledge of the external disturbance $\mathbf{d}(t)$. The rate of convergence of the observation error must be independent of initialisations $\mathbf{x}_{0}$ and $\mathbf{z}_{0}$, control input $\mathbf{U}(t)$ and unknown input $\mathbf{d}(t)$.

To simplify the convergence analysis of the DDO, it is interesting to isolate the free disturbance measurement. To do that, assume without loss of generality that $p \geq q$ with

$$
\operatorname{rank}\left[\begin{array}{l}
\mathbf{F} \\
\mathbf{G}
\end{array}\right]=q \text { and } \operatorname{rank} \mathbf{G}=q_{1} \leq q .
$$

Then, there exist an orthogonal matrix $\mathbf{V}$ and a non singular matrix $\mathbf{W}$ such that

$$
\mathbf{V}^{\mathrm{T}} \mathbf{G W}=\left[\begin{array}{cc}
\mathbf{I}_{q 1} & \mathbf{0} \\
\mathbf{0} & \mathbf{0}
\end{array}\right]
$$

According to (6), the state and the measurement equations (1)-(2) are equivalent to

$$
\begin{gathered}
\dot{\mathbf{x}}=\mathbf{A}_{1} \mathbf{x}+\sum_{i=1}^{m} \mathbf{D}_{i} \mathrm{u}_{i} \mathbf{x}+\mathbf{B} \mathbf{U}+\mathbf{F}_{1} \mathbf{y}_{1}+\mathbf{F}_{2} \mathbf{d}_{2} \\
\mathbf{y}_{1}=\mathbf{C}_{1} \mathbf{x}+\mathbf{d}_{1} \\
\mathbf{y}_{2}=\mathbf{C}_{2} \mathbf{x}
\end{gathered}
$$

where $\mathbf{A}_{1}=\mathbf{A}-\mathbf{F}_{1} \mathbf{C}_{1},\left[\begin{array}{l}\mathbf{d}_{1} \\ \mathbf{d}_{2}\end{array}\right]=\mathbf{W}^{-1} \mathbf{d}$, with $\mathbf{d}_{1} \in \mathbb{R}^{q 1}, \mathbf{d}_{2} \in \mathbb{R}^{q 2}\left(q_{1}+q_{2}=q\right),\left[\begin{array}{l}\mathbf{y}_{1} \\ \mathbf{y}_{2}\end{array}\right]=\mathbf{V}^{\mathrm{T}} \mathbf{y}$, with $\mathbf{y}_{1} \in$ $\mathbb{R}^{q 1}, \mathbf{y}_{2} \in \mathbb{R}^{v}\left(q_{1}+v=p\right),\left[\begin{array}{ll}\mathbf{F}_{1} & \mathbf{F}_{2}\end{array}\right]=\mathbf{F W}$ and $\left[\begin{array}{l}\mathbf{C}_{1} \\ \mathbf{C}_{2}\end{array}\right]=\mathbf{V}^{\mathrm{T}} \mathbf{C}$.

Without loss of generality, consider that matrix [ $\left.\begin{array}{ll}\mathbf{C} & \mathbf{G}\end{array}\right]$ is of full row rank, then $\mathbf{C}_{2}$ is of full row rank.

Note that equations (8)-(9) are obtained by multiplying (2) by $\mathbf{V}^{\mathrm{T}}$ and by using (6). Then equation (7) is deduced by inserting (8) in (1). As in Hara and Furuta (1976) and Hac (1992) we assume without loss of generality that the bilinear system (7)-(9) (or equivalently (1)-(2)) has no stable uncontrollable states with respect to the inputs $\mathbf{U}(t)$ and $\mathbf{d}(t)$ (see appendix).

\section{Disturbance Decoupled Observer Design}

By rewriting equation (2) in an equivalent form given by (8)-(9), the measurement $\mathbf{y}(t)$ has been decomposed in two parts : $\mathbf{y}_{1}(t)$ and $\mathbf{y}_{2}(t)$. $\mathbf{y}_{1}(t)$ is completely corrupted by the disturbance $\mathbf{d}_{1}(t)$. Then like $\mathbf{U}(t), \mathbf{y}_{1}(t)$ is considered as a known input in the state equation (7). $\mathbf{y}_{2}(t)$ is the disturbance-free part of the measurement $\mathbf{y}(t)$ which can be injected in the DDO in order to reconstruct the state $\mathbf{x}(t)$. Then if the system (1)-(2) is written in the form (7)-(9), the DDO (3)-(4) becomes 


$$
\begin{gathered}
\dot{\mathbf{z}}=\mathbf{H z}+\mathbf{L}_{1} \mathbf{y}_{1}+\mathbf{L}_{2} \mathbf{y}_{2}+\mathbf{J U}+\sum_{i=1}^{m} \mathbf{N}_{2, i} u_{i} \mathbf{y}_{2} \\
\hat{\mathbf{x}}=\mathbf{M z}+\mathbf{P}_{2} \mathbf{y}_{2}
\end{gathered}
$$

with [ $\left.\begin{array}{ll}\mathbf{L}_{1} & \mathbf{L}_{2}\end{array}\right]=\mathbf{L V},\left[\begin{array}{ll}\mathbf{0} & \mathbf{N}_{2, i}\end{array}\right]=\mathbf{N}_{i} \mathbf{V}$ and [ $\mathbf{0} \mathbf{P}_{2}$ ] $=\mathbf{P V}$.

3.1 Full-order Observer. For the full-order case, the state observer $\mathbf{z}(t)$ is of dimension $n$, i.e. $j=n$ and $\mathbf{M}=\mathbf{I}_{n}$. The reconstruction error between $\hat{\mathbf{x}}(t)$ in (11) and $\mathbf{x}(t)$ in (7) is given by

$$
\mathbf{e}_{f}=\hat{\mathbf{x}}-\mathbf{x}=\mathbf{z}-\mathbf{x}+\mathbf{P}_{2} \mathbf{y}_{2} \text {. }
$$

The time derivative of $\mathbf{e}_{f}(t)$ according to (7) and (11) is

$$
\begin{aligned}
\dot{\mathbf{e}}_{f}= & \mathbf{H e}_{f}+\left(\mathbf{H S}-\mathbf{S} \mathbf{A}_{1}+\mathbf{L}_{2} \mathbf{C}_{2}\right) \mathbf{x}+(\mathbf{J}-\mathbf{S B}) \mathbf{U}-\mathbf{S F}_{2} \mathbf{d}_{2} \\
& +\sum_{i=1}^{m} \mathrm{u}_{i}\left(\mathbf{N}_{2, i} \mathbf{C}_{2}-\mathbf{S D}_{i}\right) \mathbf{x}+\left(\mathbf{L}_{1}-\mathbf{S F} \mathbf{F}_{1}\right) \mathbf{d}_{1}
\end{aligned}
$$

where

$$
\mathbf{S}=\mathbf{I}_{n}-\mathbf{P}_{2} \mathbf{C}_{2}
$$

From relation (13), the following lemma gives the necessary and sufficient conditions for the observer error (13) to satisfy definition 1 (Hara and Furuta, 1976; Hac, 1992).

Lemma 1. The system (10)-(11) is a full-order DDO for the bilinear system (7)-(9) (or (1)-(2)) in accordance with definition 1 if and only if $\mathbf{H}$ is Hurwitz and the following constraints are satisfied

$$
\begin{gathered}
\mathbf{H S}-\mathbf{S A}_{1}+\mathbf{L}_{2} \mathbf{C}_{2}=\mathbf{0} \\
\mathbf{N}_{2, i} \mathbf{C}_{2}-\mathbf{S D}_{i}=\mathbf{0} \\
\mathbf{S F}_{2}=\mathbf{0} \\
\mathbf{J}=\mathbf{S B} \\
\mathbf{L}_{1}=\mathbf{S F}_{1}
\end{gathered}
$$

where $\mathbf{S}$ is given by (14).

Proof. It is easy to see that if constraints (15)-(19) are satisfied, relation (13) reduces to

$$
\dot{\mathbf{e}}_{f}=\mathbf{H e}_{f}
$$

which vanishes exponentially if and only if $\mathbf{H}$ is Hurwitz. Then (5) in definition 1 is satisfied for all $k$ since all the time derivatives of $\mathbf{e}_{f}(t)$ are governed by (20). From (13) and (14), condition (5) in definition 1 holds independently of $\mathbf{U}(t), \mathbf{d}(t), \mathbf{x}_{0}$ and $\mathbf{z}_{0}$ only if (15)-(19) are satisfied.

Note that equations (15) and (17)-(19) correspond to constraints to be satisfied for the design of DDO for linear systems (Darouach et al., 1994; Hou and Müller, 1992; Kudva et al., 1980; Kurek, 1983) and constraints (16) relate to the bilinear part. In the following, an equivalent form of constraints (16)-(17) is proposed and conditions for the existence of the DDO are deduced. This equivalent form is based on the matrix $\boldsymbol{\Phi}$ defined as

$$
\mathbf{\Phi}=\left[\begin{array}{ll}
\mathbf{F}_{2} & \mathbf{D}_{a} \mathcal{K}\left(\mathbf{C}_{2 a}\right)
\end{array}\right]
$$

and 


$$
\mathbf{d}_{a}=\left[\begin{array}{l}
\mathbf{d}_{2} \\
\mathbf{d}_{b}
\end{array}\right]
$$

where $\mathbf{D}_{a}=\left[\begin{array}{lllll}\mathbf{D}_{1} & \mathbf{D}_{2} & \ldots & \mathbf{D}_{m}\end{array}\right], \mathbf{d}_{b}(t)$ is a virtual unknown input representing the effect of bilinearities,

$$
\mathbf{C}_{2 a}=\left[\begin{array}{llll}
\mathbf{C}_{2} & \mathbf{0} & \ldots & \mathbf{0} \\
\mathbf{0} & \mathbf{C}_{2} & \ldots & \mathbf{0} \\
\ldots & \ldots & \ldots & \ldots \\
\mathbf{0} & \mathbf{0} & \ldots & \mathbf{C}_{2}
\end{array}\right] \in \mathbb{R}^{(v . m) \times(n . m)}
$$

and $\mathcal{K}(\cdot)$ spans the basis of the right-kernel of the corresponding matrix. Now, the constraints given in lemma 1 can be expressed equivalently in terms of matrix $\boldsymbol{\Phi}$.

Corollary 1. The system (10)-(11) is a full-order DDO for the bilinear system (7)-(9) (or (1)-(2)) in accordance with definition 1 if and only if $\mathbf{H}$ is Hurwitz and the following constraints are satisfied

$$
\begin{gathered}
\mathbf{H S}-\mathbf{S A}_{1}+\mathbf{L}_{2} \mathbf{C}_{2}=\mathbf{0} \\
\mathbf{N}_{2 a}=\mathbf{S D}_{a} \mathbf{C}_{2 a}^{+} \\
\mathbf{S} \boldsymbol{\Phi}=\mathbf{0} \\
\mathbf{J}=\mathbf{S B} \\
\mathbf{L}_{1}=\mathbf{S F}_{1}
\end{gathered}
$$

where $\mathbf{N}_{2 a}=\left[\begin{array}{llll}\mathbf{N}_{2,1} & \mathbf{N}_{2,2} & \ldots & \mathbf{N}_{2, m}\end{array}\right], \mathbf{S}$ is given by (14) and $\mathbf{C}_{2 a}^{+}$is a generalised inverse of $\mathbf{C}_{2 a}$ ( $\mathbf{A}^{+}$is defined by $\left.\mathbf{A}=\mathbf{A} \mathbf{A}^{+} \mathbf{A}\right)$.

Proof. The system (10)-(11) is a full-order DDO for the bilinear system (7)-(9) (or (1)-(2)) in accordance with definition 1 if and only if lemma 1 holds. Inserting equation (14) into relations (16) and (17) yields

$$
\left[\begin{array}{ll}
\mathbf{P}_{2} & \mathbf{N}_{2 a}
\end{array}\right]\left[\begin{array}{cc}
\mathbf{C}_{2} \mathbf{F}_{2} & \mathbf{C}_{2} \mathbf{D}_{a} \\
\mathbf{0} & \mathbf{C}_{2 a}
\end{array}\right]=\left[\begin{array}{ll}
\mathbf{F}_{2} & \mathbf{D}_{a}
\end{array}\right] .
$$

Since $\mathbf{C}_{2}$ is of full row rank, the matrix $\left[\mathbf{C}_{2 a}^{+} \mathcal{K}\left(\mathbf{C}_{2 a}\right)\right]$ is non-singular, then equation (25) is equivalent to

$$
\left[\begin{array}{ll}
\mathbf{P}_{2} & \mathbf{N}_{2 a}
\end{array}\right]\left[\begin{array}{cc}
\mathbf{C}_{2} \mathbf{F}_{2} & \mathbf{C}_{2} \mathbf{D}_{a} \\
\mathbf{0} & \mathbf{C}_{2 a}
\end{array}\right]\left[\begin{array}{ccc}
\mathbf{I}_{q 2} & \mathbf{0} & \mathbf{0} \\
\mathbf{0} & \mathbf{C}_{2 a}^{+} & \mathcal{K}\left(\mathbf{C}_{2 a}\right)
\end{array}\right]=\left[\begin{array}{ll}
\mathbf{F}_{2} & \mathbf{D}_{a}
\end{array}\right]\left[\begin{array}{ccc}
\mathbf{I}_{q 2} & \mathbf{0} & \mathbf{0} \\
\mathbf{0} & \mathbf{C}_{2 a}^{+} & \mathcal{K}\left(\mathbf{C}_{2 a}\right)
\end{array}\right] .
$$

Equation (26) can be rewritten as

$$
\left[\begin{array}{ll}
\mathbf{P}_{2} & \mathbf{N}_{2 a}
\end{array}\right]\left[\begin{array}{ccc}
\mathbf{C}_{2} \mathbf{F}_{2} & \mathbf{C}_{2} \mathbf{D}_{a} \mathbf{C}_{2 a}^{+} & \mathbf{C}_{2} \mathbf{D}_{a} \mathcal{K}\left(\mathbf{C}_{2 a}\right) \\
\mathbf{0} & \mathbf{I}_{v \times m} & \mathbf{0}
\end{array}\right]=\left[\begin{array}{lll}
\mathbf{F}_{2} & \mathbf{D}_{a} \mathbf{C}_{2 a}^{+} & \mathbf{D}_{a} \mathcal{K}\left(\mathbf{C}_{2 a}\right)
\end{array}\right] .
$$

Then, by using relation (21), equation (27) gives

$$
\begin{gathered}
\mathbf{N}_{2 a}=-\left(\mathbf{P}_{2} \mathbf{C}_{2}-\mathbf{I}_{n}\right) \mathbf{D}_{a} \mathbf{C}_{2 a}^{+} \\
\left(\mathbf{P}_{2} \mathbf{C}_{2}-\mathbf{I}_{n}\right) \boldsymbol{\Phi}=\mathbf{0}
\end{gathered}
$$

which are equivalent to constraints (23)-(24). Then lemma 1 and corollary 1 are equivalent. 
Since equations (16)-(17) have been expressed in a similar form as those of DDO constraints for linear systems, the following theorem can be stated.

Theorem 1. The system (10)-(11) (or (3)-(4)) is a DDO for the bilinear system (7)-(9) (or (1)-(2)) in accordance with definition 1 if and only if the following system

$$
\begin{gathered}
\dot{\mathbf{z}}=\mathbf{H z}+\mathbf{L}_{1} \mathbf{y}_{1}+\mathbf{L}_{2} \mathbf{y}_{2}+\mathbf{J U} \\
\hat{\mathbf{x}}=\mathbf{M z}+\mathbf{P}_{2} \mathbf{y}_{2}
\end{gathered}
$$

is a stable DDO for the linear system described by

$$
\begin{gathered}
\dot{\mathbf{x}}=\mathbf{A}_{1} \mathbf{x}+\mathbf{B U}+\mathbf{F}_{1} \mathbf{y}_{1}+\boldsymbol{\Phi} \mathbf{d}_{a} \\
\mathbf{y}_{1}=\mathbf{C}_{1} \mathbf{x}+\mathbf{d}_{1} \\
\mathbf{y}_{2}=\mathbf{C}_{2} \mathbf{x} .
\end{gathered}
$$

Proof. It is easy to see that if constraints in corollary 1 are satisfied, then the system (30)-(31) is a stable DDO for the linear system (32)-(34).

Based on the previous theorem, the conditions for the existence of the DDO (10)-(11) (or (3)-(4)) can be formulated in terms of invariant zeros as expressed by the following lemma.

Lemma 2. The system (10)-(11) (or (3)-(4)) is a full-order DDO for the bilinear system (7)-(9) (or (1)-(2)) if and only if the following conditions are satisfied

$$
\operatorname{rank}\left[\begin{array}{ccccc}
s \mathbf{I}_{n}-\mathbf{A} & -\mathbf{F} & -\varphi_{1} & \ldots & -\varphi_{m} \\
\mathbf{C} & \mathbf{G} & \mathbf{0} & \ldots & \mathbf{0}
\end{array}\right]=n+\operatorname{rank}\left[\begin{array}{cccc}
\mathbf{F} & \varphi_{1} & \ldots & \varphi_{m} \\
\mathbf{G} & \mathbf{0} & \ldots & \mathbf{0}
\end{array}\right], \forall s \in \mathbb{C}, \operatorname{Re}(s) \geq 0
$$

and

$$
\operatorname{rank}\left[\begin{array}{ccccc}
\mathbf{G} & \mathbf{C F} & \mathbf{C} \varphi_{1} & \ldots & \mathbf{C} \varphi_{m} \\
\mathbf{0} & \mathbf{G} & \mathbf{0} & \ldots & \mathbf{0}
\end{array}\right]=\operatorname{rank} \mathbf{G}+\operatorname{rank}\left[\begin{array}{cccc}
\mathbf{F} & \varphi_{1} & \ldots & \varphi_{m} \\
\mathbf{G} & \mathbf{0} & \ldots & \mathbf{0}
\end{array}\right]
$$

with

$$
\varphi_{i}=\mathbf{D}_{i} \mathcal{K}\left(\left[0 \quad \mathbf{I}_{p 2}\right] \mathbf{V}^{\mathrm{T}} \mathbf{C}\right) .
$$

Proof. Using theorem 1, the DDO exists if and only if the pair $\left(\mathbf{S A}_{1}, \mathbf{C}_{2}\right)$ is detectable and $\operatorname{rank} \mathbf{C}_{2} \boldsymbol{\Phi}=\operatorname{rank} \boldsymbol{\Phi}$ which are equivalent to conditions (35a) and (35b) respectively (Darouach et al. 1994). Note that the pair $\left(\mathbf{S A}_{1}, \mathbf{C}_{2}\right)$ is detectable if and only if the invariant zeros of the triple $\left(\mathbf{A}_{1}, \boldsymbol{\Phi}, \mathbf{C}_{2}\right)$ are stable (Darouach et al. 1994). Using matrices given in (6), conditions (35a) and (35b) are equivalent to

$$
\operatorname{rank}\left[\begin{array}{cc}
s \mathbf{I}_{n}-\mathbf{A}_{1} & \boldsymbol{\Phi} \\
\mathbf{C}_{2} & \mathbf{0}
\end{array}\right]=n+\operatorname{rank} \boldsymbol{\Phi}, \forall s \in \mathbb{C}, \operatorname{Re}(s) \geq 0
$$

and

$$
\operatorname{rank} \mathbf{C}_{2} \boldsymbol{\Phi}=\operatorname{rank} \boldsymbol{\Phi}
$$

respectively. 
Remark 1. Following Darouach et al. (1994), the fixed modes of the matrix $\mathbf{H}$ in (20) are the invariant zeros given by condition (35a) or (35c).

Remark 2. The necessary and sufficient conditions for the existence of the observer (without unknown input) proposed by Hara and Furuta (1976) are given by lemma 2 with $\mathbf{F}=\mathbf{0}, \mathbf{G}=\mathbf{0}$ and $\varphi_{i}=\mathbf{D}_{i} \mathcal{K}(\mathbf{C})\left(\right.$ i.e. $\mathbf{C}=\mathbf{C}_{2}$ and $\boldsymbol{\Phi}=\left[\begin{array}{lll}\boldsymbol{\varphi}_{1} & \cdots & \varphi_{m}\end{array}\right]$ in (21) if $\left.\mathbf{F}=\mathbf{0}\right)$.

3.2 Reduced-order Observer. For the reduced-order DDO $(j<n)$, the minimal order is attained when $j=n-v$. $\mathbf{C}_{2}$ being of full row rank, there exists a matrix $\mathbf{T} \in \mathbb{R}^{j \times n}$ such that

$$
\operatorname{det}\left[\begin{array}{c}
\mathbf{T} \\
\mathbf{C}_{2}
\end{array}\right] \neq 0 \text {. }
$$

Let the state of the DDO be defined by $\mathbf{z}(t)=\mathbf{T} \hat{\mathbf{x}}(t)$, the reconstruction error can be written as

$$
\mathbf{e}_{r}=\mathbf{T}(\hat{\mathbf{x}}-\mathbf{x})=\mathbf{z}-\mathbf{T} \mathbf{x} .
$$

The time derivative of $\mathbf{e}_{r}(t)$ according to (7) and (11) is

$$
\begin{gathered}
\dot{\mathbf{e}}_{r}=\mathbf{H e}_{r}+\left(\mathbf{H T}-\mathbf{T} \mathbf{A}_{1}+\mathbf{L}_{2} \mathbf{C}_{2}\right) \mathbf{x}+(\mathbf{J}-\mathbf{T B}) \mathbf{U}-\mathbf{T} \mathbf{F}_{2} \mathbf{d}_{2} \\
+\sum_{i=1}^{m} u_{i}\left(\mathbf{N}_{2, i} \mathbf{C}_{2}-\mathbf{T} \mathbf{D}_{i}\right) \mathbf{x}+\left(\mathbf{L}_{1}-\mathbf{T} \mathbf{F}_{1}\right) \mathbf{d}_{1} .
\end{gathered}
$$

Then like for the full-order case, the following lemma can be stated for the reduced-order one.

Lemma 3. The system (10)-(11) (or (3)-(4)) is a reduced-order DDO for the bilinear system (7)-(9) (or (1)-(2)) in accordance with definition 1 if and only if $\mathbf{H}$ is a stability matrix and the following constraints are satisfied

$$
\begin{gathered}
\mathbf{H T}-\mathbf{T} \mathbf{A}_{1}+\mathbf{L}_{2} \mathbf{C}_{2}=\mathbf{0} \\
\mathbf{N}_{2, i} \mathbf{C}_{2}-\mathbf{T D}_{i}=\mathbf{0} \\
\mathbf{T F}_{2}=\mathbf{0} \\
{\left[\begin{array}{c}
\mathbf{T} \\
\mathbf{C}_{2}
\end{array}\right]\left[\begin{array}{cc}
\mathbf{M} & \mathbf{P}_{2}
\end{array}\right]=\left[\begin{array}{cc}
\mathbf{I}_{n-v} & \mathbf{0} \\
\mathbf{0} & \mathbf{I}_{v}
\end{array}\right]} \\
\mathbf{J}=\mathbf{T B} \\
\mathbf{L}_{1}=\mathbf{T F}_{1} .
\end{gathered}
$$

Proof. If constraints (40)-(45) are satisfied then the observation error (39) reduces to

$$
\dot{\mathbf{e}}_{r}=\mathbf{H e}_{r}
$$

which vanishes exponentially if and only if $\mathbf{H}$ is Hurwitz. Then (5) in definition 1 is satisfied for all $k$ since all the time derivatives of $\mathbf{e}_{r}(t)$ are governed by (46).

Since $\mathbf{T} \in \mathbb{R}^{j \times n}$, equation (43) implies that relation (37) holds. Then from (11), for each pair $\left(\mathbf{z}(t), \mathbf{y}_{2}(t)\right)$, there exists an unique $\hat{\mathbf{x}}(t)$. Conversely, for each $\hat{\mathbf{x}}(t)$ in $\mathbb{R}^{n}$, there exists an unique pair $\left(\mathbf{z}(t), \mathbf{y}_{2}(t)\right)$. Then from (39), condition (5) in definition 1 holds independently of $\mathbf{U}(t), \mathbf{d}(t), \mathbf{x}_{0}$ and $\mathbf{z}_{0}$ only if (40)-(45) are satisfied. This ends the proof.

From (37), note that (43) can be equivalently rewritten as 


$$
\mathbf{M T}+\mathbf{P C}_{2}=\mathbf{I}_{n} .
$$

Using the same approach as for the full-order DDO, the necessary and sufficient constraints to be satisfied for the DDO problem can be simplified as follows.

Corollary 2. The system (10)-(11) (or (3)-(4)) is a reduced-order DDO for the bilinear system (7)(9) (or (1)-(2)) in accordance with definition 1 if and only if $\mathbf{H}$ is a stability matrix and the following constraints are satisfied

$$
\begin{gathered}
\mathbf{H T}-\mathbf{T} \mathbf{A}_{1}+\mathbf{L}_{2} \mathbf{C}_{2}=\mathbf{0} \\
\mathbf{N}_{2 a}=\mathbf{T D}_{a} \mathbf{C}_{2 a}^{+} \\
\mathbf{T} \boldsymbol{\Phi}=\mathbf{0} \\
{\left[\begin{array}{c}
\mathbf{T} \\
\mathbf{C}_{2}
\end{array}\right]\left[\begin{array}{cc}
\mathbf{M} & \mathbf{P}_{2}
\end{array}\right]=\left[\begin{array}{cc}
\mathbf{I}_{n-v} & \mathbf{0} \\
\mathbf{0} & \mathbf{I}_{v}
\end{array}\right]} \\
\mathbf{J}=\mathbf{T B} \\
\mathbf{L}_{1}=\mathbf{T F}_{1} .
\end{gathered}
$$

Proof. The system (10)-(11) is a reduced-order DDO for the bilinear system (7)-(9) (or (1)-(2)) in accordance with definition 1 if and only if lemma 3 holds. Equation (41) can be rewritten as follows

$$
\mathbf{N}_{2 a} \mathbf{C}_{2 a}=\mathbf{T D}_{a}
$$

The matrix $\left[\begin{array}{cc}\mathbf{C}_{2 a}^{+} & \mathcal{K}\left(\mathbf{C}_{2 a}\right)\end{array}\right]$ being non-singular, relation (49) is equivalent to

$$
\mathbf{N}_{2 a} \mathbf{C}_{2 a}\left[\begin{array}{ll}
\mathbf{C}_{2 a}^{+} & \mathcal{K}\left(\mathbf{C}_{2 a}\right)
\end{array}\right]=\mathbf{T D}_{a}\left[\begin{array}{ll}
\mathbf{C}_{2 a}^{+} & \mathcal{K}\left(\mathbf{C}_{2 a}\right)
\end{array}\right]
$$

which yields

and

$$
\mathbf{N}_{2 a}=\mathbf{T D}_{a} \mathbf{C}_{2 a}^{+}
$$

Then using equations (42) and (51), one obtains equation (48) which ends the proof.

Note that the reduced-order DDO constraints (40)-(45) are closed to those of the full-order DDO (see (14)-(19)) if $\mathbf{S}$ is replaced by $\mathbf{T}$ and the constraint on $\mathbf{S}$ and $\mathbf{P}_{2}$ (14) by the constraint on $\mathbf{T}, \mathbf{M}$ and $\mathbf{P}_{2}$ (43).

One can see that theorem 1 can be also applied to the reduced-order DDO. Indeed, lemma 2 gives the necessary and sufficient conditions for the existence of both reduced-order and full-order DDO for bilinear systems. Note that if only the full and the minimal-order cases have been considered here, all reduced-order cases can be straightforwardly deduced. In addition, it has been shown that, by the use of matrix $\boldsymbol{\Phi}$ (21), the design of a DDO for bilinear systems in accordance with definition 1 is reduced to the design of DDO for linear systems (see Kudva and al., 1980; Kurek, 1983; Hou and Müller, 1992; Darouach et al., 1994) which can be directly applied to the bilinear case.

Remark 3. Note that the existence of a linear DDO (i.e. with $\mathbf{N}_{2 a}=\mathbf{0}$ ) implies the existence of a bilinear DDO (i.e. with $\mathbf{N}_{2 a} \neq \mathbf{0}$ ), but the converse is not true. Indeed, if $\mathbf{N}_{2 a}=\mathbf{0}$, the constraints to be satisfied for the stability of the observation error are then given by corollaries 1 or 2 using 


$$
\boldsymbol{\Phi}=\left[\begin{array}{ll}
\mathbf{F}_{2} & \mathbf{D}_{a}
\end{array}\right]
$$

in (24), instead of matrix $\boldsymbol{\Phi}$ given in (21). Applying again theorem 1, necessary and sufficient conditions for the existence of the linear DDO for bilinear systems are given by lemma 2 with

$$
\varphi_{i}=\mathbf{D}_{i}
$$

If conditions of lemma 2 are satisfied with $\varphi_{i}$ given by (53) then these conditions are also satisfied with $\varphi_{i}$ given by (36).

\section{Application to Disturbance Decoupled Residual Observer Design}

The main idea in this paper is to transform the bilinear systems state observation problem into an equivalent one for linear systems in accordance with definition 1. This approach simplifies significantly the DDO design. It is easy to extend this approach to the design of disturbance decoupling residual observer (DDRO). As stated in Frank (1993) and in Kinnaert et al. (1995), the failure detection and isolation (FDI) problem can be formulated as a DDRO design since each residual must be sensitive to certain failure and insensitive to the other ones which can be seen as unknown inputs.

Assume that the bilinear system (7)-(9) is affected by fault vector $\mathbf{f}(t) \in \mathbb{R}^{\mu}$ as follows

$$
\begin{gathered}
\dot{\mathbf{x}}=\mathbf{A}_{1} \mathbf{x}+\sum_{i=1}^{m} \quad \mathbf{D}_{i} \mathbf{u}_{i} \mathbf{x}+\mathbf{B U}+\mathbf{F}_{1} \mathbf{y}_{1}+\mathbf{F}_{2} \mathbf{d}_{2}+\mathbf{R f} \\
\mathbf{y}_{1}=\mathbf{C}_{1} \mathbf{x}+\mathbf{d}_{1} \\
\mathbf{y}_{2}=\mathbf{C}_{2} \mathbf{x} .
\end{gathered}
$$

The aim is to design a DDRO of the following form

$$
\begin{gathered}
\dot{\mathbf{z}}=\mathbf{H z}+\mathbf{L}_{1} \mathbf{y}_{1}+\mathbf{L}_{2} \mathbf{y}_{2}+\mathbf{J U}+\sum_{i=1}^{m} \mathbf{N}_{2, i} \mathbf{u}_{i} \mathbf{y}_{2} \\
\mathbf{r}=\mathbf{M z}+\mathbf{P}_{2} \mathbf{y}_{2}
\end{gathered}
$$

such that the residual $\mathbf{r}(t)$ satisfies the following definition.

Definition 2. The system (55)-(56) is said to be a DDRO of system (54)-(8)-(9) if and only if

$$
\begin{array}{ll}
\lim _{t \rightarrow \infty} \frac{\mathrm{d}^{k}}{\mathrm{dt}^{k}} \quad \mathbf{r}(t)=\mathbf{0} \text { if } \mathbf{f}(t)=\mathbf{0} & (k=0,1,2, \ldots) \\
\lim _{t \rightarrow \infty} \frac{\mathrm{d}^{k}}{\mathrm{dt}^{k}} \quad \mathbf{r}(t) \neq \mathbf{0} \text { if } \mathbf{f}(t) \neq \mathbf{0} \quad(k=0,1,2, \ldots)
\end{array}
$$

independently of $\mathbf{U}(t), \mathbf{d}(t), \mathbf{x}_{0}$ and $\mathbf{z}_{0}$.

This definition is an extension of definition 1 to the FDI problem.

Let $\mathbf{e}_{d}(t)=\mathbf{z}(t)-\mathbf{T}_{d} \mathbf{x}(t)$ be the observation error; then the residual $\mathbf{r}(t)$ can be expressed in terms of the observation error $\mathbf{e}_{d}(t)$ and the state $\mathbf{x}(t)$

$$
\mathbf{r}=\mathbf{M e}_{d}+\left(\mathbf{P}_{2} \mathbf{C}_{2}+\mathbf{M T} \mathbf{T}_{d} \mathbf{x} .\right.
$$

Then the constraints to be fulfilled such that $\mathbf{r}(t)$ satisfies the conditions given in definition 2 are given by corollary 2 by replacing condition (43) (or (43bis)) by the following 


$$
\mathbf{P}_{2} \mathbf{C}_{2}+\mathbf{M T}_{d}=\mathbf{0} .
$$

If constraints (40)-(42), (44)-(45) (see lemma 3) and (60) hold, then the time derivative of $\mathbf{e}_{d}(t)$ is reduced to

$$
\dot{\mathbf{e}}_{d}=\mathbf{H e}_{d}-\mathbf{T}_{d} \mathbf{R f} .
$$

By using the matrix $\Phi$ given in (21), constraints (41)-(42) can be replaced by (47)-(48) (see corollary 2). Definition 2 holds since the error $\mathbf{e}_{d}(t)$ is linear.

The main difference between the DDO and the DDRO designs is that the determinant constraint (37) is not verified for DDRO since (43) is replaced by (60). But, as in the previous section, the bilinear DDRO problem in accordance with definition 2 is once again reduced to the design of a DDRO for the linear system (32)-(34) (see theorem 1) by the use of matrix $\boldsymbol{\Phi}$. For the design of a DDRO in the linear case, the reader can refer to Chang and Hsu (1993).

Note that the DDRO in the discrete-time case proposed by Yu and Shields $(1995,1996)$ and Yu et al. (1996) correspond to definition 2 since this definition implies linear observation error dynamics (see (61)). In Kinnaert et al. (1995), this is not the case since the reconstruction error dynamics is bilinear.

\section{Examples}

In this section two examples are given to illustrate the above developments. The first example concerns the quarter-car semi-active suspension worked out by Hac (1992) and the second an electromechanical actuator (Malassé et al., 1994; Rugh, 1991).

5.1 Semi-active suspension model (Hac, 1992). Consider the model of vehicle with a semi-active suspension

$\dot{\mathbf{x}}=\mathbf{A} \mathbf{x}+\mathbf{D}_{1} u_{1} \mathbf{x}+\mathbf{F} d$
where $\quad \mathbf{A}=\left[\begin{array}{cccc}0 & 1 & 0 & 0 \\ -k_{1} / m_{1} & 0 & -k_{1} / m_{1} & 0 \\ 0 & -1 & 0 & 1 \\ 0 & 0 & -k_{2} / m_{2} & 0\end{array}\right], \mathbf{F}=\left[\begin{array}{c}-1 \\ 0 \\ 0 \\ 0\end{array}\right], \mathbf{D}=\left[\begin{array}{cccc}0 & 0 & 0 & 0 \\ 0 & -1 / m_{1} & 0 & 1 / m_{1} \\ 0 & 0 & 0 & 0 \\ 0 & 1 / m_{2} & 0 & -1 / m_{2}\end{array}\right]$ and $\mathbf{C}=\left[\begin{array}{llll}1 & 0 & 1 & 0 \\ 0 & 1 & 0 & 0 \\ 0 & 0 & 1 & 0\end{array}\right]$ with $m_{1}=40[\mathrm{~kg}], m_{2}=250[\mathrm{~kg}], k_{1}=10[\mathrm{~N} / \mathrm{m}], k_{2}=5000[\mathrm{~N} / \mathrm{m}]$.

The state components are the wheel displacement relative to the road, the wheel velocity, the body displacement relative to the wheel and the body velocity. The input variable $u_{1}(t)$ is the damping ratio of an electronically controlled shock absorber and the unmeasured disturbance $d(t)$ is the rate of change of road elevation.

It is easy to see that this system satisfies the existence conditions (35a)-(36b) given in lemma 2 with $\varphi_{1}=\mathbf{D}_{1}$ (see (53)), then a linear DDO can be designed for this bilinear system. This result is closely related to the one of Hac (1992) but without computing the bilinear part of the DDO $\left(\mathbf{N}_{1}=\mathbf{0}\right)$. The design is the reduced to the pole placement of the matrix $\mathbf{H}$ using the linear DDO design given by Darouach et al. (1994) or Kurek (1983) for example. 
5.2 Electromechanical actuator (Malassé et al., 1994; Rugh, 1981). The second numerical example is an electromechanical actuator frequently used in robotics and constituting of a directcurrent motor with an elastic coupling and the load shaft as shown in the figure 1.

This plant can be described by the following bilinear state-space model

$$
\begin{gathered}
\dot{\mathbf{x}}=\mathbf{A x}+\mathbf{D}_{1} u_{1} \mathbf{x}+\mathbf{B} \mathbf{U}+\mathbf{F} d \\
\mathbf{y}=\mathbf{C} \mathbf{x}
\end{gathered}
$$

where $\quad \mathbf{A}=\left[\begin{array}{ccccc}-R_{d} L_{a} & 0 & 0 & 0 & 0 \\ 0 & -F_{m} / J_{m} & 0 & -k_{r} /\left(N J_{m}\right) & 0 \\ 0 & 1 & 0 & 0 & 0 \\ 0 & 1 / N & 0 & 0 & -1 \\ 0 & 0 & 0 & k_{r} / J_{c} & -F_{c} / J_{c}\end{array}\right], \mathbf{x}=\left[\begin{array}{c}i_{a} \\ \omega_{m} \\ \theta_{m} \\ \Delta \Gamma \\ \omega_{c}\end{array}\right], \mathbf{F}=\left[\begin{array}{c}0 \\ 0 \\ 0 \\ 0 \\ -1 / J_{c}\end{array}\right]$,

$$
\mathbf{D}_{1}=\left[\begin{array}{ccccc}
0 & -k_{d} / L_{a} & 0 & 0 & 0 \\
k_{d} J_{m} & 0 & 0 & 0 & 0 \\
0 & 0 & 0 & 0 & 0 \\
0 & 0 & 0 & 0 & 0 \\
0 & 0 & 0 & 0 & 0
\end{array}\right], \mathbf{B}=\left[\begin{array}{cc}
0 & 1 / L_{a} \\
0 & 0 \\
0 & 0 \\
0 & 0 \\
0 & 0
\end{array}\right], \mathbf{C}=\left[\begin{array}{lllll}
1 & 0 & 0 & 0 & 0 \\
0 & 0 & 1 & 0 & 0 \\
0 & 0 & 0 & 0 & 1
\end{array}\right] \text { and } \mathbf{U}=\left[\begin{array}{c}
i_{e} \\
v_{a}
\end{array}\right]=\left[\begin{array}{l}
u_{1} \\
u_{2}
\end{array}\right] \text {. }
$$

The state variables are the armature current $i_{a}(t)$, the motor shaft velocity $\omega_{m}(t)$, the motor shaft angular position $\theta_{m}(t)$, the angular rotation $\Delta \Gamma(t)$ between the motor shaft and the load shaft due to the elastic coupling and the load shaft angular velocity $\omega_{c}(t)$. The control inputs are the stator current $i_{e}(t)$ and the armature voltage $v_{d}(t)$. The unknown input $d(t)$ is the torque due to the Coulomb frictions and the load reactions. In the state space description, $J_{m}$ and $J_{c}$ represent the motor and the load shaft inertia, $F_{m}$ and $F_{c}$ the motor and the load viscous friction coefficients, $k_{a}$ the motor torque constant, $k_{r}$ the coupling rigidity coefficient and $N$ the gear ratio. The numerical values of these parameters are $\mathrm{N}=$ $20, R_{a}=1[\Omega], L_{a}=0.05[\mathrm{H}], k_{a}=0.156\left[\mathrm{~m}^{2} \mathrm{kgsec}^{-2} \mathrm{~A}^{-2}\right], k_{r}=37.7\left[\mathrm{~m}^{2} \mathrm{kgsec}^{-2}\right], F_{m}=0.0032\left[\mathrm{~m}^{2}\right.$ $\left.\mathrm{kgsec}^{-1}\right], F_{c} \cong 0\left[\mathrm{~m}^{2} \mathrm{kgsec}^{-1}\right], J_{m}=2.4 \mathrm{e}^{-4}\left[\mathrm{~m}^{2} \mathrm{~kg}\right], \mathrm{J}_{c}=0.0825\left[\mathrm{~m}^{2} \mathrm{~kg}\right]$.

Note that since the output equation is not affected by the unknown input, the output transformation (6) is not necessary (i.e. $\mathbf{C}=\mathbf{C}_{2}$ and $\mathbf{F}=\mathbf{F}_{2}$ ). It can be shown that a linear DDO cannot be designed since conditions (35a)-(35b) given in lemma 2 are not satisfied with $\varphi_{1}=\mathbf{D}_{1}$ (see (53)) :

$$
\text { the invariant zero of the triple }\left(\mathbf{A},\left[\begin{array}{ll}
\mathbf{F} & \mathbf{D}_{1}
\end{array}\right], \mathbf{C}\right) \text { is }\{0\}
$$

and

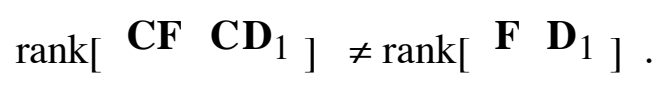

Now, conditions (35a)-(35b) given in lemma 2 are satisfied with $\varphi_{1}=\mathbf{D}_{1} \mathcal{K}(\mathbf{C})$ (see (36)) since :

the triple $\left(\mathbf{A},\left[\mathbf{F} \quad \mathbf{D}_{1} \mathcal{K}(\mathbf{C})\right], \mathbf{C}\right)$ has no invariant zero

and

$$
\left.\operatorname{rank}\left[\mathbf{C F} \mathbf{C D}_{1} \mathcal{K}(\mathbf{C})\right]_{]}=\operatorname{rank}_{[} \mathbf{F} \mathbf{D}_{1} \mathcal{K}(\mathbf{C})\right]_{]}=2 .
$$

Then, a reduced-order bilinear DDO can be designed by the approach proposed for linear systems by Kudva et al. (1980) or Hou and Müller (1992). The matrices of this DDO ((3)-(4)) are

$$
\mathbf{M}=\left[\begin{array}{ll}
0 & 0 \\
1 & 0 \\
0 & 0 \\
0 & 1 \\
0 & 0
\end{array}\right], \mathbf{P}=\left[\begin{array}{ccc}
1 & 0 & 0 \\
0 & 286.6667 & 0 \\
0 & 1 & 0 \\
0 & -2.4964 & 0 \\
0 & 0 & 1
\end{array}\right], \mathbf{H}=\left[\begin{array}{cc}
-300 & -7854.2 \\
2.5464 & 0
\end{array}\right], \mathbf{L}=\left[\begin{array}{ccc}
0 & -66393 & 0 \\
0 & 729.9735 & -1
\end{array}\right],
$$


$\mathbf{J}=\left[\begin{array}{ll}0 & 0 \\ 0 & 0\end{array}\right], \mathbf{N}_{1}=\left[\begin{array}{cccc}650 & 0 & 0 \\ 0 & 0 & 0\end{array}\right]$, with $\mathbf{T}=\left[\begin{array}{lllll}0 & 1 & -286.6667 & 0 & 0 \\ 0 & 0 & 2.4964 & 1 & 0\end{array}\right]$ and $\boldsymbol{\Phi}=\left[\begin{array}{rrr}0 & -3.12 & 0 \\ 0 & 0 & 0 \\ 0 & 0 & 0 \\ 0 & 0 & 0 \\ -12.1212 & 0 & 0\end{array}\right]$.

In figures 2 and 3 are displayed the actual value (solid line) and the estimated value (dashed line) of the unmeasured state components $\omega_{m}(t)$ and $\Delta \Gamma(t)$ respectively.

\section{Conclusion}

The main objective of this paper has been the characterisation of a class of bilinear systems subjected to unknown disturbances for which there exists a DDO with linear error dynamics. By the use of an instrumental matrix $\boldsymbol{\Phi}$, it was shown that the design of this kind of DDO is equivalent to the design of DDO for a linear system. Necessary and sufficient conditions for the existence of full-order and reduced-order DDO are then easily deduced in terms of invariant zero. This result simplifies significantly the DDO design with regard to those proposed by Hac (1992) and Saif (1993) since synthesis of DDO for linear systems proposed in the literature can be applied directly. Using the properties of the matrix $\boldsymbol{\Phi}$, this approach is extended to the characterisation of a class of bilinear systems for which there exists a linear DDO and to the fault detection of bilinear systems. These results have been illustrated for a vehicle semi-active suspension and an electromechanical actuator.

\section{References}

Bornard G., Couenne N., Celle F., 1988, "Regularly persistent observers for bilinear systems", Lecture Notes in Control and Information Sciences, New Trends in Nonlinear Control Theory, pp. 130-140.

Chang S.K., Hsu P.L., 1993, "Fault detection observer design for linear systems with unknown inputs", Proceedings, 2nd European Control Conference, Groningen.

Darouach M., Zasadzinski M., Xu S.J., 1994, "Full-order observers for linear systems with unknown inputs”, IEEE Transactions on Automatic Control, vol. 39, pp. 606-609.

Frank P.M., 1993, “Advances in observer-based fault diagnosis", Proceedings, International Conference on Fault Diagnosis, TOOLDIAG'93, Toulouse.

Funahashi Y., 1979, "Stable state estimator for bilinear systems", International Journal of Control, vol. 29, pp. 181-188.

Grasselli O.M., Isidori A., 1981, "An existence theorem for observers of bilinear systems”, IEEE Transactions on Automatic Control, vol. AC-26, pp. 1299-1300.

Hac A., 1992, "Design of disturbance decoupled observer for bilinear systems", Transactions of the ASME, Journal of Dynamic Systems, Measurement, and Control, vol. 114, pp. 556-562.

Hara S., Furuta K., 1976, "Minimal order state observers for bilinear systems", International Journal of Control, vol. 24, pp. 705-718. 
Hou M., Müller P.C., 1992, "Design of observers for linear systems with unknown inputs", IEEE Transactions on Automatic Control, vol. 37, pp. 871-875.

Kinnaert M., Peng Y., Hammouri H., 1995, “The fundamental problem of residual generation for bilinear systems up to output injection", Proceedings, 3rd European Control Conference, Roma.

Kudva P., Viswanadham N., Ramakrishna A., 1980, "Observers for linear systems with unknown inputs", IEEE Transactions on Automatic Control, vol. 25, pp. 113-115.

Kurek J., 1983, "The state reconstruction for linear systems with unknown inputs", IEEE Transactions on Automatic Control, vol. 36, pp. 511-515.

Malassé O., Zasadzinski M., Iung C., Hayar M., Darouach M., 1994, “Hळ design using normalized coprime factors : an application to an electromechanical actuator", Proceedings, $3 r d$ IEEE Conference on Control Applications, Glasgow.

Mohler R.R., 1991, Nonlinear systems, Volume II, Applications to bilinear control, Prentice Hall.

Rugh W.J., 1981, Nonlinear system theory : The Volterra/Wiener approach, The Johns Hopkins University Press.

Saif M., 1993, "A disturbance accomodating estimator for bilinear systems", Proceedings, American Control Conference, San Francisco.

Williamson D., 1977, "Observation of bilinear systems with application to biological control", Automatica, vol. 13, pp. 243-254.

Yu D., Shields D.N., 1995, "Fault diagnosis in bilinear systems - A survey", Proceedings, 3rd European Control Conference, Roma.

Yu D., Shields D.N., 1996, “A bilinear fault detection observer”, Automatica, vol. 32, pp. 15971602.

Yu D., Shields D.N., S. Daley, 1996, “A bilinear fault detection observer and its application to a hydraulic drive system", International Journal of Control, vol. 64, pp. 1023-1047.

\section{APPENDIX}

The bilinear system (7)-(9) has some stable uncontrollable states with respect to the inputs $\mathbf{U}(t)$ and $\mathbf{d}(t)$ when equation (7) can be decomposed as follows (Hara and Furuta, 1976; Hac, 1992)

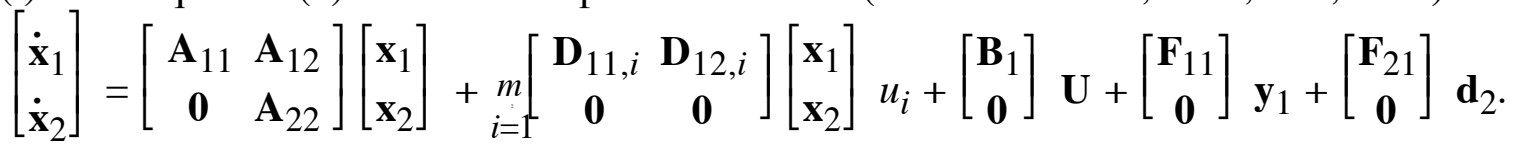

According to (A.1), equations (8) and (9) can be rewritten as

$$
\begin{gathered}
\mathbf{y}_{1}=\mathbf{C}_{11} \mathbf{x}_{1}+\mathbf{C}_{12} \mathbf{x}_{2}+\mathbf{d}_{1} \\
\mathbf{y}_{2}=\mathbf{C}_{21} \mathbf{x}_{1}+\mathbf{C}_{22} \mathbf{x}_{2} .
\end{gathered}
$$

As pointed out by Hara and Furuta (1976) and Hac (1992), an observer may exist for the bilinear system (A.1)-(A.3), even if the Sylvester equations (15) or (40) do not hold. But, in this case, a necessary condition is that the matrix $\mathbf{A}_{22}$ is Hurwitz. Indeed if lemma 1 or 3 are satisfied except for (15) or (40) which are replaced by 


$$
\mathbf{H T}-\mathbf{T} \mathbf{A}_{1}+\mathbf{L}_{2} \mathbf{C}_{2}=\left[\begin{array}{cc}
\mathbf{0} & \mathbf{0} \\
\mathbf{0} & \boldsymbol{\Xi}_{22}
\end{array}\right]
$$

where $\boldsymbol{\Xi}_{22} \neq \mathbf{0}$ is a matrix of appropriate dimension ( $\mathbf{T}$ is replaced by $\mathbf{S}$ for the full-order case), then the time derivative of the reconstruction error $\mathbf{e}(t)$ (13) or (39) can be rewritten as

$$
\dot{\mathbf{e}}=\mathbf{H e}+\left[\begin{array}{cc}
\mathbf{0} & \mathbf{0} \\
\mathbf{0} & \boldsymbol{\Xi}_{22}
\end{array}\right]\left[\begin{array}{c}
\mathbf{0} \\
\mathbf{x}_{2}
\end{array}\right] \text {. }
$$

The observation error (A.5) with $\boldsymbol{\Xi}_{22} \neq \mathbf{0}$ (i.e. when (15) or (40) do not hold) verifies condition (5) in definition (1) since the dynamics of the uncontrollable state $\mathbf{x}_{2}$ is linear and exponentially stable (i.e. $\mathbf{A}_{22}$ is Hurwitz) and $\mathbf{H}$ is Hurwitz.

$\mathbf{A}_{22}$ being an Hurwitz matrix, the DDO design with $\boldsymbol{\Xi}_{22}=\mathbf{0}$ yields no loss of generality since, for the bilinear system (A.1)-(A.3), there exists a DDO if and only if the constraints (15)-(19) for the fullorder case or the constraints (40)-(45) for the reduced-order case can be solved. Indeed, according to the partition of $\mathbf{x}(t)$ in (A.1) and choosing the observer matrices as follows

$$
\begin{gathered}
\mathbf{H}=\left[\begin{array}{cc}
\mathbf{H}_{11} & \mathbf{H}_{12} \\
\mathbf{0} & \mathbf{H}_{22}
\end{array}\right], \mathbf{T}=\left[\begin{array}{cc}
\mathbf{T}_{11} & \mathbf{T}_{12} \\
\mathbf{0} & \mathbf{I}
\end{array}\right], \mathbf{M}=\left[\begin{array}{cc}
\mathbf{M}_{11} & \mathbf{M}_{12} \\
\mathbf{0} & \mathbf{I}
\end{array}\right], \mathbf{N}_{2, i}=\left[\begin{array}{c}
\mathbf{N}_{21, i} \\
\mathbf{0}
\end{array}\right], \mathbf{P}_{2}=\left[\begin{array}{c}
\mathbf{P}_{21} \\
\mathbf{0}
\end{array}\right], \\
\mathbf{L}_{1}=\left[\begin{array}{c}
\mathbf{L}_{11} \\
\mathbf{0}
\end{array}\right], \mathbf{L}_{2}=\left[\begin{array}{c}
\mathbf{L}_{21} \\
\mathbf{0}
\end{array}\right] \text { and } \mathbf{J}=\left[\begin{array}{c}
\mathbf{J}_{1} \\
\mathbf{0}
\end{array}\right]
\end{gathered}
$$

with $\mathbf{H}_{11}$ and $\mathbf{H}_{22}$ Hurwitz (for the full-order case, $\mathbf{M}_{11}=\mathbf{I}, \mathbf{M}_{12}=\mathbf{0}$ and $\mathbf{T}$ is replaced by $\mathbf{S}$ ) and using relation (A.4) yields

$$
\mathbf{H}_{22}=\mathbf{A}_{22}+\boldsymbol{\Xi}_{22} \text {. }
$$

Then a DDO of $\mathbf{x}(t)$ satisfying lemmas 1 or $3\left(\boldsymbol{\Xi}_{22}=\mathbf{0}\right)$ can be always designed in three steps since $\mathbf{A}_{22}$ is Hurwitz.

Step 1. Set

$$
\mathbf{H}_{22}=\mathbf{A}_{22} \text {. }
$$

Step 2. Determine matrices $\mathbf{H}_{11}, \mathbf{T}_{11}, \mathbf{L}_{21}, \mathbf{M}_{11}$ and $\mathbf{P}_{21}$ by designing a DDO of $\mathbf{x}_{1}(t)$ for system (A.1)-(A.3) by setting $\mathbf{x}_{2}(t)=\mathbf{0}$.

Step 3. (i) Determine $\mathbf{T}_{12}$ by solving $\quad \mathbf{N}_{21, i} \mathbf{C}_{22}-\mathbf{T}_{12} \mathbf{D}_{12, i}=\mathbf{0}$.

(ii) Set

$$
\mathbf{M}_{12}=-\left[\begin{array}{l}
\mathbf{T}_{12} \\
\mathbf{C}_{22}
\end{array}\right]\left[\begin{array}{l}
\mathbf{T}_{11} \\
\mathbf{C}_{21}
\end{array}\right] \text {. }
$$

(iii) Set

$$
\mathbf{H}_{12}=\mathbf{T}_{11} \mathbf{A}_{12}+\mathbf{T}_{12} \mathbf{A}_{22}-\mathbf{H}_{11} \mathbf{T}_{12}-\mathbf{L}_{21} \mathbf{C}_{22}
$$

Note that (i) in step 3 may always been solved even if $\boldsymbol{\Xi}_{22} \neq \mathbf{0}$ (i.e. when (15) or (40) do not hold) since this constraint is deduced from (16) or (41). In step 2, the constraint (37) holds by replacing $\mathbf{T}$ and $\mathbf{C}_{2}$ by $\mathbf{T}_{11}$ and $\mathbf{C}_{21}$ respectively, then $\mathbf{M}_{12}$ can be obtained by (ii) in step 3.

For the full order case, $\mathbf{T}_{11}$ and $\mathbf{T}_{12}$ are replaced by $\mathbf{S}_{11}$ and $\mathbf{S}_{12}$ respectively. In step 3, (i) becomes $\mathbf{S}_{12}=-\mathbf{P}_{21} \mathbf{C}_{22}$ and (ii) is reduced to $\mathbf{M}_{12}=\mathbf{0}$.

Step 1 yields $\boldsymbol{\Xi}_{22}=\mathbf{0}$ in (A.4), guaranteeing the Sylvester equations (15) or (40) hold. But if $\mathbf{H}_{22}$ is an Hurwitz such that $\mathbf{H}_{22} \neq \mathbf{A}_{22}$ (i.e. $\boldsymbol{\Xi}_{22} \neq \mathbf{0}$ ), then the observation error $\mathbf{e}(t)$ in (A.5) satisfies the condition (5) in definition 1 since the uncontrollable state $\mathbf{x}_{2}(t)$ is generated by a stable autonomous linear process (see (A.1)). 
Remark. The bilinear systems (1)-(2) and (7)-(9) have the same uncontrollable states since, using the notations in (A.1)-(A.3) with relation (6), matrices in (1)-(2) are given by

$$
\begin{gathered}
\mathbf{A}=\left[\begin{array}{cc}
\mathbf{A}_{11}+\mathbf{F}_{11} \mathbf{C}_{11} & \mathbf{A}_{12}+\mathbf{F}_{11} \mathbf{C}_{12} \\
\mathbf{0} & \mathbf{A}_{22}
\end{array}\right], \mathbf{F}=\left[\begin{array}{cc}
\mathbf{F}_{11} & \mathbf{F}_{21} \\
\mathbf{0} & \mathbf{0}
\end{array}\right] \mathbf{W}^{-1}, \\
\mathbf{C}=\mathbf{V}\left[\begin{array}{ll}
\mathbf{C}_{11} & \mathbf{C}_{12} \\
\mathbf{C}_{21} & \mathbf{C}_{22}
\end{array}\right] \text { and } \mathbf{G}=\mathbf{V}\left[\begin{array}{cc}
\mathbf{I}_{q 1} & \mathbf{0} \\
\mathbf{0} & \mathbf{0}
\end{array}\right] \mathbf{W}^{-1} .
\end{gathered}
$$

\section{Figures}

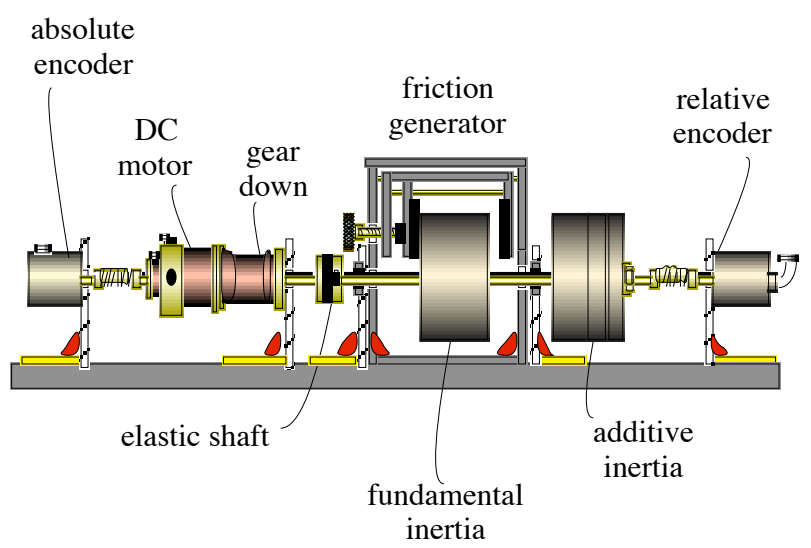

Figure 1: electromechanical actuator

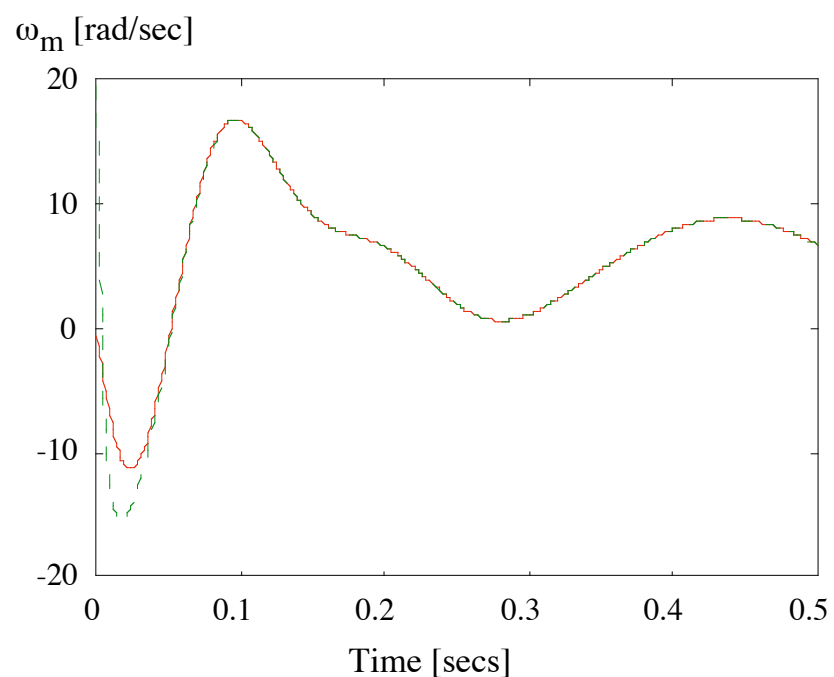

Figure 2: motor shaft velocity $\omega_{m}(t):$ actual value (solid line), estimated value (dashed line) 


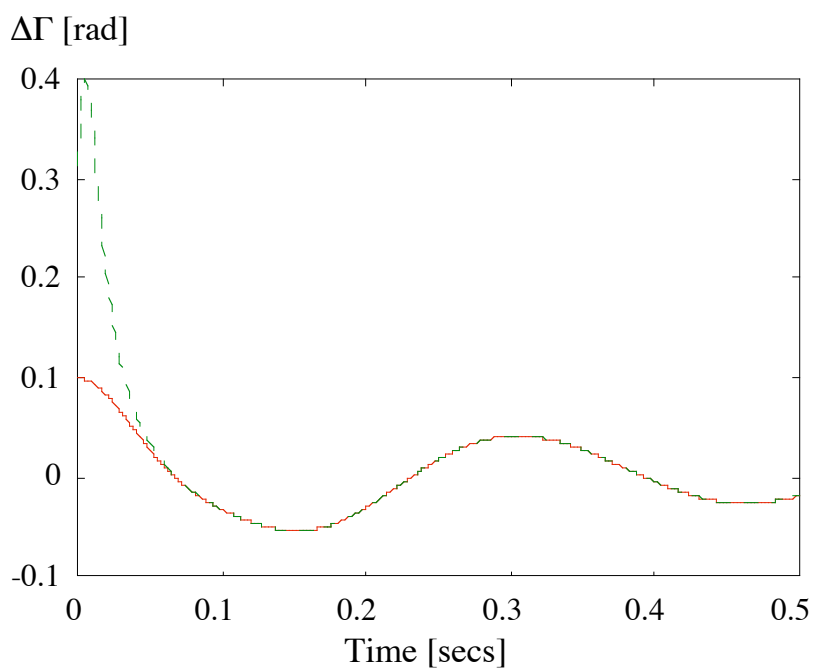

Figure 3: angular rotation $\Delta \Gamma(t)$ : actual value (solid line), estimated value (dashed line) 